\title{
Foreign body response induced by tissue expander implantation
}

\author{
LINGLING SHENG ${ }^{*}$, QINGXIONG YU*, FENG XIE and QINGFENG LI
}

\begin{abstract}
Department of Plastic and Reconstructive Surgery, Shanghai 9th People's Hospital, Shanghai Jiao Tong University, School of Medicine, Shanghai 200011, P.R. China
\end{abstract}

Received June 27, 2013; Accepted December 12, 2013

DOI: $10.3892 / \mathrm{mmr} .2013 .1881$

\begin{abstract}
The foreign body response (FBR) is described as the host's response to implanted biomaterials, which involves a complex cascade of immune modulators. The dynamic changes of immune cells, inflammatory cytokines and the formation of a fibrous capsule remain to be elucidated. In the present study, a model of subcutaneous implantation of a tissue expander was used. The results revealed that macrophages, the main immune cells in FBR, infiltrated into the expanded tissue and located at the tissue-material interface from day 1-90. Following the decrease of the number of macrophages, collagen deposited and fibroblasts transformed into myofibroblasts at the tissue-material interface, leading to the formation of a fibrous capsule from day 14. The persistent existing macrophages led to a high expression of proinflammatory cytokines, including tumor necrosis factor- $\alpha$ and interleukin-1 $\beta$, both of which initiated the NK- $\mathrm{BB}$ and JNK inflammatory pathways, mediating the FBR to tissue expander implantation.
\end{abstract}

\section{Introduction}

Tissue expansion is one of the most significant innovations of the 20th century in plastic surgery (1). It is a process which involves implanting a silicon sac subcutaneously and regularly injecting saline into it, resulting in the formation of new skin under the mechanical stretch and providing a supply of tissue similar in color, structure and adnexal distribution to the adjacent skin in order to offer highly effective repair. Therefore, tissue expansion is an extremely useful technique and has a wide range of applications in plastic surgery, particularly for defective repair following large scar resection (2).

Correspondence to: Professor Qingfeng Li, Department of Plastic and Reconstructive Surgery, Shanghai 9th People's Hospital, Shanghai Jiao Tong University, School of Medicine, 639 Zhizaoju Road, Shanghai 200011, P.R. China

E-mail: dr.liqingfeng@shsmu.edu.cn

*Contributed equally

Key words: tissue expander, silicon, foreign body response, fibrous capsule, macrophages
The tissue expanders are mainly composed of silicone and following implantation into the body, will initiate a host response named the foreign body response (FBR). This leads to the formation of a fibrous capsule around the expander in order to 'seal it off' (3). In the clinic, the thick fibrous capsule formed around the implant prolongs the period of tissue expansion and makes the expanded skin contract markedly. Thus, 4-6 months are always required to achieve the needed expansion; in addition, limited expanded skin could be harvested and used in a repair surgery in a period of treatment and re-expansion is required. The length of time required for the expansion is disadvantageous since it causes inconvenience in the daily lives of the patients. In addition, it increases the incidence of complications, including infections, rupture and exposure of the tissue expander.

Due to the wide application of the tissue expander, it is of high diagnostic and clinical significance to clarify the exact molecular mechanisms behind the phenomenon of peri-silicone implant capsule formation. In other words, the identification of a method to inhibit the formation of the fibrous capsule may provide a novel therapy to accelerate tissue expansion and to harvest more regenerative tissue. In order to provide more specific information with regard to the immune cells and cytokines, known to be critical to the progress of FBR, the present study reports their dynamic change by a subcutaneous implantation model. We hypothesize that this knowledge is essential as it would offer a proper intervention time to decrease the harmful effects of the FBR on the application of tissue expanders in the clinic.

\section{Materials and methods}

Ethics statement. All the animal procedures were approved under the guidelines of Shanghai Jiao Tong University Medical Center, the Institutional Animal Care and Use Committee (Shanghai, China).

Implant model and tissue collection. A tissue expansion model was used to analyze the dynamic changes of the immune cells and cytokines. A total of 20 Lewis rats (six weeks old; male; body weight, 110-120 g; Shanghai Experimental Animal Center, Shanghai, China) were anesthetized with $3 \%$ sodium pentobarbital at $0.13 \mathrm{ml} / 100 \mathrm{~g}$ and shaved. A single incision was made at the back of rats, and a subcutaneous pocket of $3.0 \times 6.0 \mathrm{~cm}$ was created into which the tissue expander was placed. The expander pot was set under the head skin. Next, 
$15 \mathrm{ml}$ physiological saline was injected through these pots and an additional volume of $3 \mathrm{ml}$ was injected into the expander pocket once a week.

Identification of immune cells in peri-implant tissue. The immune cells in peri-implant tissue were identified using immunohistochemistry. The tissues harvested on day 1, 3, 7 14, 28 and 90 post-implantation were fixed and embedded in paraffin for a histological assay. Following blocking with hydrogen peroxide $\left(\mathrm{H}_{2} \mathrm{O}_{2}\right)$ and with a protein blocking agent, the primary antibodies, mouse anti-rat CD68, CD4 and CD11c (Abcam, Cambridge, UK) were applied directly at $4^{\circ} \mathrm{C}$ overnight. Subsequent to washing in phosphate-buffered saline (PBS), the sections were incubated in phycoerythrin-coupled anti-mouse antibody (DAKO, Glostrup, Denmark) for $1 \mathrm{~h}$ at $37^{\circ} \mathrm{C}$ and the cell nuclei were counterstained with hematoxylin. The control samples were processed following the same protocol but with the omission of the primary antibody.

Fibrous capsule and collagen deposition. Hematoxylin-eosin (HE) staining was applied to observe the fibrous capsule formed around the tissue expander. Collagen deposition in the tissue around the implant was studied by Masson's trichrome staining. Briefly, they were fixed in preheated Bouin's solution at $56^{\circ} \mathrm{C}$ for $15 \mathrm{~min}$, then in Harris' hematoxylin for $5 \mathrm{~min}$ and stained with Masson's trichrome stain (trichrome stain LG solution; Sigma-Aldrich, St. Louis, MO, USA) for $5 \mathrm{~min}$. The stained sections were observed for collagen deposition by bright field microscopy. The $\alpha$-smooth muscle actin (SMA) positive cells in the peri-implant tissue were stained by immumohistochemical staining with anti- $\alpha$-SMA antibody (Abcam) as described in the aforementioned method.

Protein level of cytokines. For detection of growth factors, $0.5 \mathrm{~g}$ tissue from the above area was collected from each group $(n=4$ for each group). The tissues were homogenized in $500 \mu \mathrm{l}$ tissue protein extraction reagent (CWBIO, Beijing, China) and $5 \mu 1$ phenylmethanesulfonyl fluoride (Sigma-Aldrich). Subsequent to centrifugation at $11,176 \mathrm{x}$ g for $10 \mathrm{~min}$, the supernatant was collected for the assay of interleukin (IL)-1 $\alpha$, IL-1 $\beta$, IL-2, IL-4, IL-13, monocyte chemoattractant protein-1 (MCP-1), interferon- $\gamma($ IFN- $\gamma$ ) and tumor necrosis factor (TNF)- $\alpha$ using a Protein Quantibody array kit according to the manufacturer's instructions (R\&D Systems, Minneapolis, MN, USA). The signals can be visualized through the use of a laser scanner equipped with a Cy3 wavelength (Thermo Fisher, Waltham, MA, USA). The data extraction can be performed with the majority of the microarray analysis software (GenePix, ScanArray Express, ArrayVision or MicroVigene).

Western blotting for $N F-\kappa B, J N K$ and P38 MAPK. In order to determine the levels of NF- $\kappa \mathrm{B}, \mathrm{JNK}$ and p38 MAPK, nuclear extracts were prepared from the expanded tissue and were totally resolved on $10 \%$ SDS-PAGE. Following electrophoresis, the proteins were electrotransferred onto nitrocellulose filters, probed with rabbit polyclonal Abs against NF- $\mathrm{B}, \mathrm{JNK}$ and p38 MAPK (Cell Signaling Technology, Inc., Boston, MA, USA), and detected by chemiluminescence (ECL, Amersham, Piscataway, NJ, USA). The bands obtained were quantitated with Personal Densitometer Scan version 1.30 using Image
Quant software version 3.3 (Molecular Dynamics, Inc., Sunnyvale, CA, USA).

\section{Results}

Infiltration of immune cells. The macrophages revealed a persistent infiltration, and mainly located at the tissue-material interface. The number of macrophages increased steadily around the tissue expander since day 1, and the highest amount of positive cells were observed in the tissue at day 7. Next, the number decreased gradually until day 90 (Fig. 1). However, there were a few $\mathrm{CD}^{+}$lymphocytes and $\mathrm{CD} 11 \mathrm{c}^{+}$dendritic cells observed (data not shown).

Formation of the fibrous capsule at the tissue-material interface. HE staining revealed that fibrous tissue began to form around the tissue expander at day 14, increased to maximum at 30 days and decreased gradually with a persistent thin layer at day 90. Masson's trichrome staining revealed the most evident deposition of collagen around the implants at day 14, which maintained with a gradual decrease over time until day 90 . It has been previously reported that myofibroblasts contributed to walling off the foreign body in chronic inflammation and were transformed from fibroblasts in this process (4). In the present study, a few of $\alpha$-SMA positive myofibroblasts were present around silicone at 14 and 30 days and a decrease was observed at 90 days (Fig. 2).

Cytokines in peri-implant tissue. Among all these inflammatory cytokines, TNF- $\alpha$ revealed the highest expression and IL-1 $\beta$ and IL-13 moderately increased in the expanded tissue at all the time points when compared with the tissue which was without an implantation of a tissue expander. The level of the cytokines IL- $1 \alpha$, IL- 4 , IFN- $\gamma$ and MCP- 1 was almost at similar levels to that in the normal tissue (Fig. 3).

Expression of $N F-\kappa B, J N K$ and $p 38 M A P K$. In order to gain an improved understanding of the potential mechanisms underlying the initiation of the inflammatory response by inflammatory cytokines, western blotting of NF- $\kappa \mathrm{B}, \mathrm{JNK}$ and p38 MAPK was performed. As revealed in the present study, NF- $\kappa \mathrm{B}$ and JNK were expressed from day 1-90 in the peri-implant tissue, and p38 MAPK did not show any difference in expression when compared with the control group (Fig. 3).

\section{Discussion}

Silicone implants are widely used in the field of plastic surgery for wound repair and cosmetic augmentation. The formation of a thick fibrous capsule around the implant limits its potential maximum effect. The implantation of a tissue expander and injury caused by the surgical procedure triggers a tissue reaction, including the secretion of pro-inflammatory cytokines and chemoattractants. As a result, an inflammatory response is caused and infiltration of macrophages, neutrophils and lymphocytes to the site of the implantation.

The macrophage, which is the dominant cell in FBR, mainly located at the tissue/material interface throughout the time points. It has been revealed that cytokines including the transforming growth factor- $\beta$, platelet derived growth factor, 


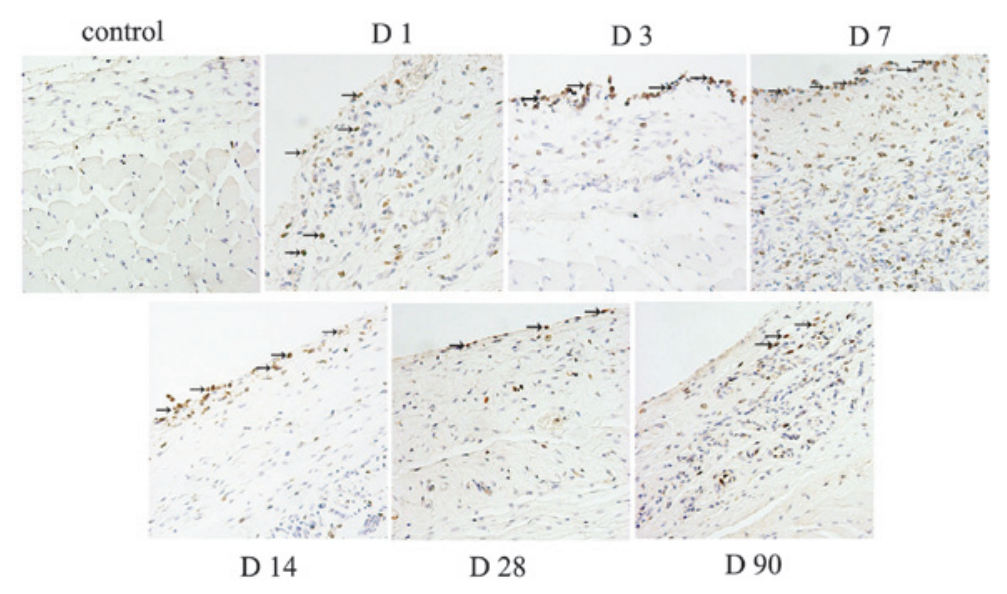

Figure 1. Macrophages infiltrated into the tissue at all time points and mainly located at the tissue/material interface. There was an evident increase of the number of macrophages at the seventh day, after which the number of cells decreased, but persisted until day 90 . There was no positive staining observed in the normal tissue (magnification, x200; arrows show CD68-positive cells).

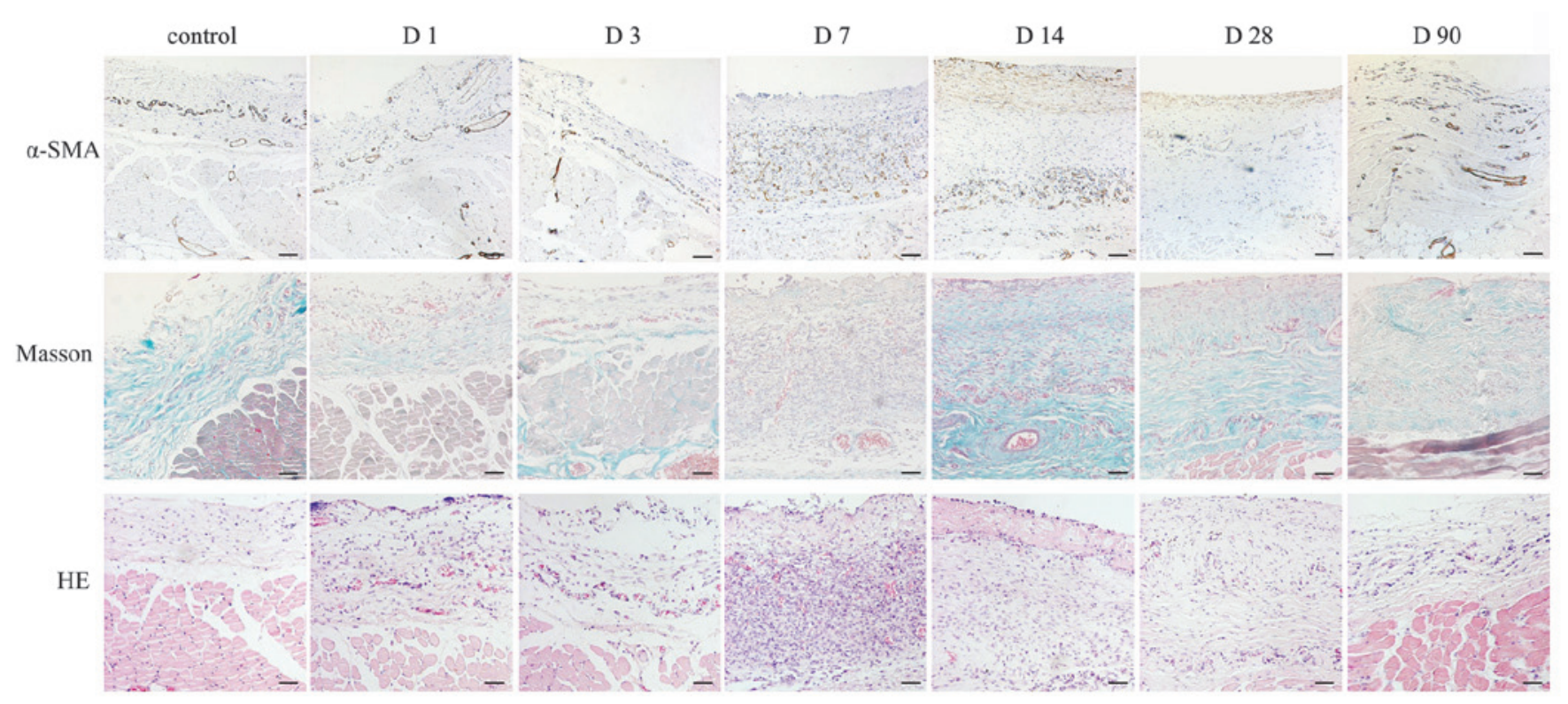

Figure 2. There was an evident appearance of $\alpha$-SMA positive cells in the covering tissue and an evident deposition of collagen at day 14 , along with the formation of a fibrous capsule around the tissue expander (top, middle and bottom, respectively). Then, the $\alpha$-SMA positive cells and collagen deposition decreased and persisted till day 90. $\alpha$-SMA, smooth muscle actin.

chemokine (C-X-C motif) ligand 4 (CXCL4) and IL-1 in tissues could direct macrophages to the wound site (5). A previous study indicated that mast cell degranulation and the release of histamine may play an integral role in recruiting macrophages to the implantation site (6). Next, infiltrated macrophages could secrete more inflammatory cytokines and chemokines, including MCP-1, granulocyte and granulocyte-macrophage colony stimulating factors, attracting more macrophages to the wound site, and engaging in the subsequent events of the FBR (7-9). However, in the present study, there was no difference observed in the expression of MCP-1. This is consistent with a previous study in which it was demonstrated that the recruitment of monocytes to subcutaneous implant sites was not affected by MCP-1 (10).

Previously, it has been demonstrated that besides the macrophages, $\mathrm{CD} 4^{+} \mathrm{T}$ lymphocytes also participate in the FBR and are located at the tissue/material interface (4). Rodriguez et $a l$ hypothesized that they are present in the FBR due to adaptive immunity (11). However, in the present study, there was no positive staining observed. This is contradictory with the study by Joseph et al (4) which reported $\mathrm{CD} 4^{+} \mathrm{T}$ lymphocytes appeared around silicone expander implants. The presence of lymphocytes has been demonstrated to promote macrophage adhesion via paracrine effects and direct signaling $(12,13)$. The absence of the proinflammatory cytokine IFN- $\gamma$ in the present study correlates with the lack of the Th1 type inflammatory response observed. During FBR, Th2-polarized T cells were originally hypothesized to be the source of the cytokines IL-10, IL-4 and IL-13 $(12,14)$. However, it was demonstrated that macrophage- or neutrophil-derived cells may serve as a source of IL-13 at the beginning of inflammation and also maintain IL-13 production during the chronic inflammatory response to 
A

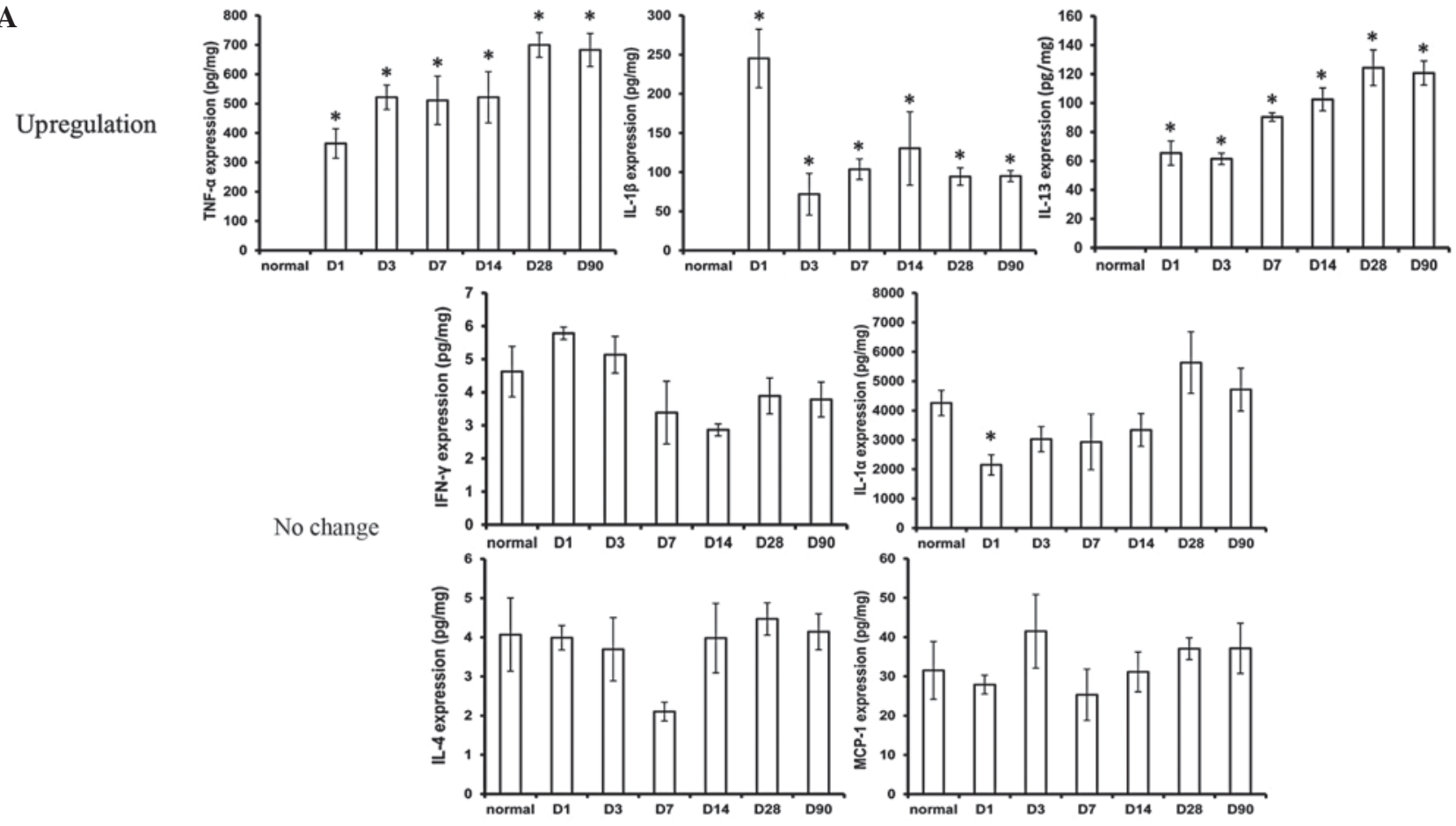

B

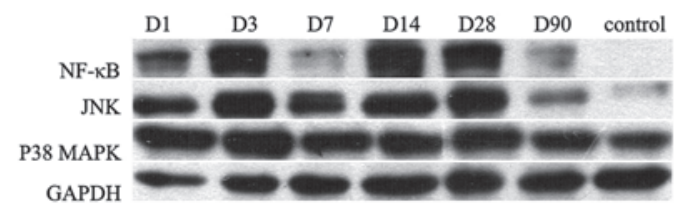

Figure 3. Expression of inflammatory cytokines and pathways initiated by implantation of a tissue expander in the covering tissue. (A) The expression of TNF- $\alpha$ increased the most evidently, and IL-1 $\beta$ and IL-13 also increased at all the time points, and IL- $1 \alpha$, IL-4, IFN- $\gamma$ and MCP-1 revealed no change in the covering tissue after implantation of a tissue expander when compared with the normal tissue $\left({ }^{*} \mathrm{P}<0.05\right)$. (B) Western blotting showing that $\mathrm{NF}-\mathrm{KB}$ and $\mathrm{JNK}$ were triggered until day 90, while P38 MAPK revealed no evident change. TNF- $\alpha$, tumour necrosis factor- $\alpha$; IL, interleukin.

the biomaterial $(15,16)$. In other words, $C D 4^{+} \mathrm{T}$ lymphocytes did not affect the secretion of IL-13. It has been shown that IL-4 and IL-13 play significant roles in determining the extent and degree of the subsequent development of the FBR. In the present study, moderately expressed IL-13 demonstrated more important roles than IL-4 due to IL-4 not showing significant changes. Besides the macrophages and $\mathrm{CD}^{+} \mathrm{T}$ lymphocytes, there was a lack of expression of CD11c in the covering tissue (data not shown), indicating that dendritic cells do not play a significant role in the progress of FBR in vivo, which is consistent with a previous study (3). However, in a previous in vitro study, it was demonstrated that biomaterials can affect the maturation of dendritic cells (17).

In the present study, the macrophages predominated over the first two weeks, then the cells decreased, followed by an accumulation of collagen and myofibroblasts around the implant resulting from cytokines released by the macrophages. This led to the formation of a fibrous capsule around the implant from the second week. The main cell types found in fibrotic tissue are fibroblasts and myofibroblasts. Transformation of fibroblasts to phenotypically different myofibroblasts occurs in FBR (4). An in vitro experiment with silicone particles by Granchi et al has led to a hypothesis that the macrophage ingestion of these particles induces a state of activation leading to the release of cytokines with high fibrogenic activity (18). In the present study, $\alpha$-SMA-positive myofibroblasts appeared at day 14 and 28, accompanied by the deposition of collagen.

As the main source of inflammatory cytokines, the persistent existence of macrophages caused the continuous high expression of TNF- $\alpha$ and IL-1 $\beta$. It has been proven that pro-inflammatory cytokines, including TNF- $\alpha$ and IL-1 $\beta$, could initiate the inflammatory response through the main inflammatory pathways, including JNK, p38 MAPK and $\mathrm{NF}-\kappa \mathrm{B}(19-22)$. JNK and NF- $\mathrm{B}$ are preferentially activated by cytokines, growth factors or cellular damage, and p38 MAPK is potently activated by environmental stress (23). Activation of these pathways triggers downstream signaling cascades that lead to the production of pro-inflammatory cytokines which are important mediators of acute and chronic inflammation, FBR and cell apoptosis $(24,25)$. In addition, a previous study has reported that TNF- $\alpha$ expression was associated with an increased Baker grade of periprosthetic capsular contracture following breast implant surgery, and positive TNF- $\alpha$ staining in breast capsules was localized to fibroblasts, macrophages and were extracellularly close to the prosthesis (26).

A number of published models have provided a basic cellular and cytokine signaling knowledge with regard to FBR development. However, to the best of our knowledge, there 
are no published studies examining the dynamic changes of the immune cells and inflammatory cytokines caused by implantation of the tissue expander. The requirement to study the change lies in finding an appropriate time point to inhibit the immune reaction in order to further interfere with the formation of the fibrous capsule. In the clinic, it is of great significance to gain such an understanding and the reduction of the thickness of the fibrous capsule may be a novel direction to accelerate the process of tissue expansion.

\section{Acknowledgements}

This study was supported by grants from the National Natural Science Foundation of China (no. 30730092 and 30925034) and the National Key Project of Scientific and Technical Supporting Programs Funded by the Ministry of Science \& Technology of China (no. 2012BAI11B03).

\section{References}

1. Radovan C: Breast reconstruction after mastectomy using the temporary expander. Plast Reconstr Surg 69: 195-208, 1982.

2. Sheng L, Yang M, Du Z, Yang Y and Li Q: Transplantation of stromal vascular fraction as an alternative for accelerating tissue expansion. J Plast Reconstr Aesthet Surg 66: 551-557, 2013.

3. Higgins DM, Basaraba RJ, Hohnbaum AC, Lee EJ, Grainger DW and Gonzalez-Juarrero M: Localized immunosuppressive environment in the foreign body response to implanted biomaterials. Am J Pathol 175: 161-70, 2009.

4. Joseph J, Mohanty M and Mohanan PV: Role of immune cells and inflammatory cytokines in regulation of fibrosis around silicone expander implants. J Mater Sci Mater Med 21: 1665-76, 2010.

5. Broughton G 2nd, Janis JE and Attinger CE: The basic science of wound healing. Plast Reconstr Surg 117: 12S-34S, 2006.

6. Tang L, Jennings TA and Eaton JW: Mast cells mediate acute inflammatory responses to implanted biomaterials. Proc Natl Acad Sci USA 95: 8841-6, 1998.

7. Seo Y and Murakami M: Monitoring of intracellular ammonium in perfused rat salivary gland by nitrogen-14 nuclear magnetic resonance spectroscopy. Proc Biol Sci 244: 191-6, 1991.

8. Brodbeck WG, Nakayama Y, Matsuda T, Colton E, Ziats NP and Anderson JM: Biomaterial surface chemistry dictates adherent monocyte/macrophage cytokine expression in vitro. Cytokine 18: 311-319, 2002.

9. Brodbeck WG, Voskerician G, Ziats NP, Nakayama Y, Matsuda T and Anderson JM: In vivo leukocyte cytokine mRNA responses to biomaterials are dependent on surface chemistry. J Biomed Mater Res A 64: 320-329, 2003.

10. Kyriakides TR, Foster MJ, Keeney GE, Tsai A, Giachelli CM, Clark-Lewis I, Rollins BJ and Bornstein P: The CC chemokine ligand, CCL2/MCP1, participates in macrophage fusion and foreign body giant cell formation. Am J Pathol 165: 2157-2166, 2004.
11. Rodriguez A, Voskerician G, Meyerson H, MacEwan SR and Anderson JM: T cell subset distributions following primary and secondary implantation at subcutaneous biomaterial implant sites. J Biomed Mater Res A 85: 556-565, 2008.

12. Brodbeck WG, Macewan M, Colton E, Meyerson H and Anderson JM: Lymphocytes and the foreign body response: lymphocyte enhancement of macrophage adhesion and fusion. J Biomed Mater Res A 74: 222-229, 2005.

13. Chang DT, Colton E and Anderson JM: Paracrine and juxtacrine lymphocyte enhancement of adherent macrophage and foreign body giant cell activation. J Biomed Mater Res A 89: 490-498, 2009.

14. Anderson JM, Rodriguez A and Chang DT: Foreign body reaction to biomaterials. Semin Immunol 20: 86-100, 2008.

15. Brandt E, Woerly G, Younes AB, Loiseau S and Capron M: IL-4 production by human polymorphonuclear neutrophils. J Leukoc Biol 68: 125-130, 2000.

16. Woerly G, Lacy P, Younes AB, Roger N, Loiseau S, Moqbel R and Capron M: Human eosinophils express and release IL-13 following CD28-dependent activation. J Leukoc Biol 72: 769-779, 2002.

17. Yoshida M, Mata J and Babensee JE: Effect of poly(lactic-co-glycolic acid) contact on maturation of murine bone marrow-derived dendritic cells. J Biomed Mater Res A 80: 7-12, 2007.

18. Granchi D, Cavedagna D, Ciapetti G, Stea S, Schiavon P, Giuliani R and Pizzoferrato A: Silicone breast implants: the role of immune system on capsular contracture formation. J Biomed Mater Res 29: 197-202, 1995

19. Manna SK and Aggarwal BB: Vesnarinone suppresses TNF-induced activation of NF-kappa B, c-Jun kinase, and apoptosis. J Immunol 164: 5815-5825, 2000.

20. Ogata M: p38 MAP kinase in the immune response. Tanpakushitsu Kakusan Koso 47: 2261-2267, 2002 (In Japanese).

21. Karin $M$ and Ben-Neriah Y: Phosphorylation meets ubiquitination: the control of NF-[kappa]B activity. Annu Rev Immunol 18: 621-663, 2000.

22. Zandi E, Rothwarf DM, Delhase M, Hayakawa M and Karin M: The IkappaB kinase complex (IKK) contains two kinase subunits, IKKalpha and IKKbeta, necessary for IkappaB phosphorylation and NF-kappaB activation. Cell 91: 243-252, 1997.

23. Ho PJ, Chou CK and Yeh SF: Role of JNK and p38 MAPK in Taiwanin A-induced cell death. Life Sci 91: 1358-1365, 2012.

24. Huh JE, Kang KS, Chae C, Kim HM, Ahn KS and Kim SH: Roles of $\mathrm{p} 38$ and JNK mitogen-activated protein kinase pathways during cantharidin-induced apoptosis in U937 cells. Biochem Pharmacol 67: 1811-1818, 2004.

25. Kang HJ, Soh Y, Kim MS, Lee EJ, Surh YJ, Kim HR, Kim SH and Moon A: Roles of JNK-1 and p38 in selective induction of apoptosis by capsaicin in ras-transformed human breast epithelial cells. Int J Cancer 103: 475-482, 2003.

26. Tan KT, Wijeratne D, Shih B, Baildam AD and Bayat A: Tumour necrosis factor- $\alpha$ expression is associated with increased severity of periprosthetic breast capsular contracture. Eur Surg Res 45: 327-332, 2010. 